\title{
The Extension Decision Analysis Method based on Object-element Transformation
}

\author{
Xiao Xiaonan \\ Xiamen University Tan Kah Kee College \\ Zhangzhou, Fujian, China \\ xiaoxn@xujc.com
}

\begin{abstract}
In order to solve the problem of incompatibility in the real world, it must be based on dialectical thinking to develop a unique and creative means and methods compatible with incompatible ones. Therefore, in this paper, by means of mathematical tools and extension set matter-element transformation analysis method, the extension set are discussed deeply the practical background and its relationship with the classic set, fuzzy set, on the basis of further discussed from the philosophical foundation and mathematical system extension set is not empty, extension domain can be changed by mapping, transformation incompatible problems for incompatible logical foundation and theoretical basis, and then seek the law to solve the problem of incompatibility and extension decision analysis method, to achieve the modernization of the enterprise and the best economic benefits for enterprises to provide the best scientific decision.
\end{abstract}

Keywords-Extension set; Object-element transformation; Incompatibility; Best scientific decision

\section{INTRODUCTION}

With the deepening of mankind's understanding of nature and society, scientific decision-making and control has become the core of modern enterprise production and management. The use of mathematical tools to understand the world and transform the world into a new period. There are many factors involved in the production and management of enterprises and the decision-making problems of complex and large systems, the constraints are also complex. And there are a lot of incompatibility problems. To this, traditional decision theory and mathematical method have been impotent [1-3]. In order to solve the problem of incompatibility in the real world, it must be based on dialectical thinking to develop a unique and creative means and methods compatible with incompatible ones. Therefore, in this paper, by means of mathematical tools and extension set matter-element transformation analysis method, the extension set are discussed deeply the practical background and its relationship with the classic set, fuzzy set, on the basis of further discussed from the philosophical foundation and mathematical system extension set is not empty, extension domain can be changed by mapping, transformation incompatible problems for incompatible logical foundation and theoretical basis, and then seek the law to solve the problem of incompatibility and extension decision analysis method, to achieve the modernization of the enterprise and the best economic benefits for enterprises to provide the best scientific decision [4-6]. In-depth research shows that the extension decision analysis method based on physical element transformation is a powerful tool and decision method to solve the problem of incompatibility in economic science and objective world.

\section{A NEW WAY OF THINKING -- THE INCOMPATIBLE PROBLEM IS THE COMPATIBLE EXTENSION METHOD}

We know that the logical basis of the classical collection is the exclusion law of the binary logic, which shows the relationship between the one and the other. And fuzzy set performance is a kind of logical relation, fuzzy logic, which is also one of them here, there is no law of excluded middle, the range of its study fuzzy field of boundary is not clear, it is the object of study is to seek a close to the level to further depict the nature of things, from the fuzzy to find out the high level of accuracy.

Fuzzy set theory and the classical set theory, though, is only describes the contradictions of objective things on both sides of the differences, basically solve the problem of compatibility, feasible solution is to seek the solution set, or judge is incompatible problems, points out its solution, and can not describe under certain conditions can be transformed each other, the two sides in particular intermediary transition can be to the transformation of the two sides phenomenon. In fact, the incompatibility problem does not equal neither solution nor invariable. From the dialectical point of view, the incompatible problem can also be converted to be compatible with the change of the condition. The "extension set" can be used to describe this kind of contradictory transformation phenomenon It is one of the theoretical pillars of the meta-analysis. Matterelement analysis to things can turn the rule as the research object, and try to put the law of thinking to solve the problem of incompatibility of standardization and formalization and mathematically, this is a new system of mathematics development, its prospect is very considerable.

Unlike the classical set and fuzzy sets, the philosophical basis of extension sets is:

(1)The element chi has a property rho (such as qualified finished product);

(2)The element does not have the property of rho (such as waste); 
(3)The element is changed from a non-property rho to a property of rho (such as rework);

(4)The rho of the element is rho without the property of rho (such as semiconductor).

It can be seen that the extension set is divided into three parts: the classical domain, the non-domain and the extension domain. The elements in the extension domain do not have certain properties, but under certain conditions, they can be changed into a certain property, so that the extension can be transformed by mapping and transformation can not be described as compatible.

In nature and real life there are many is not things, a lot of the fact that the law of excluded middle of classical mathematics only adapted to the certain scope, beyond this range, can appear paradox. However, in the sense of extension set, Russell's paradox will not appear.

Thus, the set of "not its own elements" in Russell's paradox is a critical element of $\mathrm{Q}$. It's both belong to the nature of the $\mathrm{Q}$, and does not belong to the nature of the $\mathrm{Q}$, that is to say, in the sense of extension set, there will be no Russell paradox, which laid a does exist in the objective world is not only things and non mathematical basis, explain the extension set is not empty.

The above explanation is that the difference between the extension set and the classical set and the fuzzy set is that it is under certain conditions " $\mathrm{x} \notin \mathrm{A}$ can transform into $\mathrm{x} \in \mathrm{A}$ ". The transformation relations of things and to use extension operation rule, it enhances the practicability of extension set, and established on the basis of extension matter-element decision method, will certainly to promote the transformation and scientific description of incompatible problems objective practical aspects for us to develop a new way of thinking and way, thus to solve the economic science and the objective world in the incompatible problems in all kinds of macro and micro decision-making to provide optimal decision and satisfactory solution.

\section{THE TRANSFORMATION ANALYSIS METHOD FOR THE TRANSFORMATION OF HYDRAULIC VALVE}

The following application element transformation method is close to the saccharification process of the beer production process.

Bottles of a certain institute winery in the second phase expansion project in 2016, brewing workshop saccharification process to improve the automation level of production, the guangdong some hydraulic parts factory of hydraulic pump, hydraulic valve of saccharifying pot renovation for number 3 . In the scope of work permitted by the original, the physical elements of the hydraulic valve work are described as follows:

Hydraulic valve work pressure $20 \mathrm{~kg}$, Valve daily oil leakage $50 \mathrm{~kg}$;
Number of valve springs 1 to 30 times, Valve opening range 0 to $50 \mathrm{~mm}$;

\section{Valve oil seal use times 1 to 2 times.}

If in this process, spring break, often at least a year need to replace thousands of spring, and often piston seal ring oil, hydraulic oil consumption 15 tons, still need several mechanic maintenance. The results not only can not improve the production automation level, but also often have serious running oil accidents. For this, the factory sent staff to the valve manufacturer to inquire, but did not get the specific feasible method for the valve.

To this end, the plant nanxian is engaged in scientific and technological research. By repeated trial run in scientific research, found that if they do not transform the form a complete set of hydraulic valve, in order to reduce fuel consumption, reduce the spring break is impossible, it's a incompatible problem.

In order to realize the desire of local automation, it is necessary to adopt the method of object element analysis to transform the hydraulic valve and the incompatible problem is the compatible problem. According to the principle of hydraulic drive, when the valve is the main part of the valve, the spring is in constant pressure, and the elastic limit must be broken when it exceeds the elastic limit. Accordingly, according to the characteristics of the supporting process, the spring has been shrunk to make the spring have buffer space in the cylinder, thus reducing the impulse pressure and avoiding the fracture. Secondly, we removed the upper oil seal ring from the piston to the oil hole, and then the piston moved to the oil seal to prevent oil leakage. The material elements of the modified new process are described as follows:

After the renovation work pressure 10 to $20 \mathrm{~kg}$, Valve daily oil leakage 0 ;

Number of valve springs More than 300 times, Valve opening range 0 to $25 \mathrm{~mm}$;

\section{Valve oil seal use times More than 300 times}

Due to the adoption of the meta-analysis method, the appropriate transformation is obtained and the incompatible problem is transformed into a compatible problem. However, due to the reduction of the scope of the process, the material time is extended. In order to solve the incompatible problem, we according to the spring can shorten enough to generate forces the pistons to return to the length of the initial displacement in situ, further analysis, adopted the thickening piston transformation, makes the distance between two oil seal ring is extended, so as to increase the open area (30-35 mm), get the ideal matching process, avoid the waste and accidents in the process of beer production, improves the production automation level, reduce the production cost, a year for the country save a lot of money. 


\section{CONCLUSION AND OUTLOOK}

The classic set, fuzzy set, and the successive generation of extension sets gradually make up for the lack of people's understanding of things, and make people understand the problem continuously. Determine on the fuzzy set membership function make the brain thinking from the traditional binary logic to multi-valued logic, and correlation functions are established in the extension set so people can study from the quality and quantity at the same time, which makes the results to solve the problem of incompatible quantitative, promoted the incompatible problems satisfactorily resolved, opens up to allow a contradiction under the premise of logic, the dialectical logic combined with a form of the extension logic.

Economic science suggests that the development of contemporary economic science has expanded from the study of things to the study of the complex large system, from the single numerical research and development to a variety of numerical composite research, from the qualitative or quantitative research development of single to complex qualitative and quantitative research, it is not only expanded the research scope from the inevitable phenomenon to accidental phenomena, from accurate phenomenon is extended to the fuzzy phenomenon, but also further research emerge in large Numbers in the objective world of incompatible problem [7-8]. And extension decision is based on the matter-element transformation can with its extensive research object and the research methods of unique gradually formed its own set of research theory and method, in order to further develop the idea of people to provide a new approach of ideal decision.

\section{REFERENCES}

[1] PENG H Y, RUAN L Z, XIANG J L. A note on boundary layer of a nonlinear evolution system with dam $\pi$ ng and diffusions[J]. J Math Anal Appl, 2015, 426:1099-1129

[2] YAO L, ZHANG T ,ZHU C J. Boundary layers for compressible Navier-Stokes equations with density-dependent viscosity and cylindrical symmetry [J]. Ann Inst H Poincare Anal Non Lineaire, 2011, 28:677-709.

[3] XUE L. Well-posedness and zero micro-rotation viscosity limit of the 2D micropolar fluid equtions[J]. Math Meth Appl Sci, 2011,34:17601777 .

[4] COMELLAS F, SAMPELS M.Statistical mechanics and its applications[J]. Physica A ,2002,309:231-235.

[5] ISAACS I M.Finite group theory [M]. Providence, Rhode Island :American Mathematical Society, 2011:1-354

[6] YANG Y,YANG Q.Singular values of nonnegative rectangular tensor [J]. Front Math China, 2011,(2):363-378.

[7] JIANG S ,ZHANG J W ,ZHAO J N .Boundary-layer efforts for the 2-D Boussinesq equations with vanishing diffusivity limit in the half plane [J].J Diff Eqns, 2011,250:3907-3936.

[8] RUAN L Z ,ZHU C J.Boundary layer for nonlinear evolution equations with damping and diffusion $[\mathrm{J}]$. Discrete Contin Dyn Syst Ser A, 2012,32:331-352. 